\title{
TITLE:
}

\section{Clinical characteristics and risk factors of ocular candidiasis.}

\section{$\operatorname{AUTHOR}(S)$ :}

Nagao, Miki; Saito, Takashi; Doi, Shoichi; Hotta, Gou; Yamamoto, Masaki; Matsumura, Yasufumi; Matsushima, Aki; Ito, Yutaka; Takakura, Shunji; Ichiyama, Satoshi

\section{CITATION:}

Nagao, Miki ...[et al]. Clinical characteristics and risk factors of ocular candidiasis.. Diagnostic microbiology and infectious disease 2012, 73(2): 149-152

\section{ISSUE DATE:}

2012-06

URL:

http://hdl.handle.net/2433/156158

\section{RIGHT:}

(c) 2012 Elsevier Inc.; This is not the published version. Please cite only the published version.; この論文は出版社版でありません。引用の際に は出版社版をご確認ご利用ください。 
1 Clinical characteristics and risk factors of ocular

2 candidiasis.

4 Miki Nagao ${ }^{1,2,3}$, Takashi Saito ${ }^{1,2}$, Shoichi Doi ${ }^{3,4}$, Gou Hotta ${ }^{1,2}$,

5 Masaki Yamamoto1,2, Yasufumi Matsumura ${ }^{1,2}$, Aki Matsushima, ${ }^{1,2}$,

6 Yutaka Ito ${ }^{1,5}$, Shunji Takakura ${ }^{1,2}$, Satoshi Ichiyama ${ }^{1,2}$

7

81 Department of Infection Control and Prevention

9 Kyoto University Hospital, Kyoto, Japan

112 Department of Clinical Laboratory Medicine,

12 Kyoto University Graduate School of Medicine, Kyoto, Japan

143 Department of Infectious Disease

15 Kyoto Katsura Hospital, Kyoto, Japan

16

174 Hematology Division of Internal Medicine

18 Kyoto Katsura Hospital, Kyoto, Japan 
5 Department of Respiratory Medicine

21 Kyoto University Graduate School of Medicine, Kyoto, Japan

22

23 Running title

24 Clinical characteristics of ocular candidiasis

25

26 Corresponding author

$27 \quad$ Miki Nagao

28 Department of Infection Control and Prevention

29 Kyoto University Hospital

$30 \quad$ Tel \& Fax $+81-75-751-4967$

3154 Shogoin-Kawahara-cho,

32 Sakyo-ku, Kyoto, 6068507 Japan

33 Email: mnagao@kuhp.kyoto-u.ac.jp 


\section{Abstract}

35 Ocular candidiasis is a major complication of Candida

36 bloodstream infection (BSI). This study was performed to reveal

37 clinical characteristics of ocular candidiasis. Of the 220 patients

38 with Candida BSI, 204 cases received ophthalmology

39 consultations between January 2005 and December 2011 at two

40 teaching hospitals. Fifty-four (26.5\%) cases had findings

41 consistent with the diagnosis of ocular candidiasis. Of these 54

42 cases, $43(79.6 \%)$ were diagnosed within 7 days after a positive

43 blood culture. Among ocular candidiasis cases, more cases were

44 due to Candida albicans (P = 0.034 OR; 3.68 95\% CI 1.11-12.2)

45 and had higher beta-D-glucan values $(\mathrm{P}=0.001 \mathrm{OR} ; 9.99$ 95\% CI

$462.60-21.3)$. We need to consider fundoscopic examination to be

47 performed within first 7 days of therapy, especially for those

48 patients who have $C$. albicans BSIs and higher beta-D-glucan

49 values. Additionally, follow-up fundoscopic examination should

50 be considered before stopping therapy for high-risk patients. 
51

52

\section{Introduction}

Bloodstream infections (BSIs) caused by Candida species have been reported to be increasingly frequent in recent decades, possibly due to rapid changes in medical practice. Candida BSI can lead to hematogenous dissemination and metastatic ocular infection with potentially devastating consequences.

Consequently, a rise in related mortality and prolonged hospitalisation has been reported [Edmond et al., 1999; Jarvis et al., 1995; Kao et al., 1999; Pfaller and Diekema, 2007; Rentz et al., 1998; Sheng et al., 2005; Wisplinghoff et al., 2004].

Normally, patients who have chorioretinitis alone are often asymptomatic and respond better to systemic antifungal therapy than those with vitreal involvement. However, in advanced stages, the intravitreal injection of an antifungal agent with or without vitrectomy is needed. Thus, it is very important for doctors to properly diagnose ocular candidiasis in the early stages of the infection.

In this study, patients with blood cultures positive for Candida BSIs were reviewed for the incidence and clinical characteristics of ocular candidiasis to reveal the risk factors of 
ocular candidiasis.

\section{Materials and Methods}

\section{Study design}

This study was performed at two teaching hospitals in Kyoto, Japan. Kyoto University Hospital (KUH) is a tertiary care university hospital with 1240 beds, and Katsura Hospital is an emergency hospital with 585 beds. Infectious disease physicians perform proactive interventions for all patients with BSI in these hospitals. In cases of Candida BSIs, catheter removal is recommended, blood cultures are collected to confirm all negative results, and finally, fundoscopy is performed by ophthalmologists usually within first 7 days of therapy. Candida BSI was defined by at least one positive blood culture for Candida species and a clinical sign of infection (e.g., fever, hypotension or tachypnea). Two hundred and twenty cases of Candida BSIs were diagnosed in the two Kyoto teaching hospitals from January 2005 to December 2011. To assess the incidence and clinical characteristics of patients with ocular involvement, we performed medical chart reviews of the Candida BSI patients who had 
91

92

93

94

consulted ophthalmologists. For the classification of ocular candidiasis, we incorporated the criteria proposed by Oude Lashof [Oude Lashof et al., 2011]. Proven ocular candidiasis was defined as ocular lesions in combination with positive histology or a positive culture of a vitreous aspirate. Probable Candida endophthalmitis was defined as vitritis or fluffy lesions with extensions into the vitreous humour. Probable Candida chorioretinitis was defined as deep focal white infiltrates in the retina. If signs of chorioretinitis were observed in patients with an underlying systemic disease that reportedly exhibits similar lesions (e.g., diabetes, hypertension or concomitant bacteremia), these cases were classified as possible ocular candidiasis.

Clinical information acquired from medical charts included age, sex, underlying diseases, receipt of corticosteroids or other immunosuppressive agents during the previous 30 days, any antimicrobial therapy during the previous 30 days, surgery during the previous 30 days, time to first negative blood culture, interval between blood culture and antifungal therapy, interval between sign of infection and removal of the catheter or antifungal agents, interval between positive fungal culture and 
111 catheter removal, the specific fungal species, antifungal therapy

112 and 30-day mortality. Digestive tract involvement included any

113 gastrointestinal disorders such as malignancies of digestive tract

114 and inflammatory bowel diseases. The (1,3)-8-D-glucan (BDG)

115 test values that were taken within 3 days after positive blood

116 cultures were also evaluated. At Katsura, the BDG values were

117 determined using the Fungitec G test (Seikagaku Corporation,

118 Tokyo, Japan). At KUH, the BDG values were determined using

119 the WAKO B-glucan test (Wako Pure Chemical Industries, Tokyo,

120 Japan). The results were analysed according to the

121 manufacturer's instructions.

\section{Statistical analysis}

123 Categorical variables were compared using Fisher's exact test.

124 Continuous variables were compared using the Kruskal-Wallis

test or the Mann-Whitney U test. BDG values under the limit of

126 detection were considered to be $0.0 \mathrm{pg} / \mathrm{mL}$. Receiver-operating

127 characteristic (ROC) curves for the BDG levels were constructed,

128 and their optimal cut-off values were determined with the

129 maximum Youden index. Potential factors associated with

130 ocular candidiasis were examined by Cox proportional hazards 
131 regression analysis. All covariates with a $p$-value of less than

1320.10 on univariate analyses were subjected to further selection by

133 the above-mentioned multivariate analyses. The data were

134 analysed with PASW software version 18.0 (SPSS) for Microsoft

135 Windows. All $\mathrm{P}$ value tests were two-tailed, and $\mathrm{P}<0.05$ was

136 considered statistically significant.

137

138 Results

139 Incidence

140 Of the 220 patients with Candida BSI, 204 presented to

141 ophthalmologists for the diagnosis of ocular candidiasis were

142 included in this study. Six of the 16 Candida BSI patients who

143 did not consult ophthalmologists included critically ill patients

144 whose prognosis had been presumed to be very poor or who died

145 before the identification of positive fungal cultures.

146 Fifty-four (26.5\%) of the 204 Candida BSI patients who were

147 evaluated by ophthalmologists had fundoscopic abnormalities

148 that met the criteria for ocular candidiasis. Among ocular

149 candidiasis cases, 10 were probable endophthalmitis, 24 were

150 probable chorioretinitis, and 20 cases were possible 
151 chorioretinitis.

\section{$152 \quad$ Epidemiologic characteristics}

153 The baseline characteristics of the study population are shown in

154 Table 1. The groups with or without ocular involvement did not

155 differ with respect to age, sex, diabetes mellitus status, the use of

156 immunosuppressive agents or the use of systemic antibiotics

157 within the previous month, but more patients with ocular

158 involvement had malignancies. In addition, more patients with

159 ocular manifestations had digestive tract abnormalities (e.g.,

160 digestive tract surgery, inflammatory bowel syndromes,

161 malignancy of a digestive tract), whereas ocular candidiasis was

162 rare in the departments of Dermatology, Rheumatology and

163 Cardiovascular Surgery. Ocular candidiasis patients were

164 infected significantly more frequently with Candida albicans and

165 less often with $C$. parapsilosis than patients without retinal

166 lesions. The length of time to the first negative blood culture,

167 the time to catheter removal and the administration of antifungal

168 agents did not differ between groups.

169 Timing of fundscopic examination

170 One hundred and eighty (88.2\%) patients received fundscopic 
171 examination once and 24 patients received twice or more.

172 Ocular abnormalities consistent with ocular candidiasis were

173 diagnosed within 7 days after positive blood culture in 43 patients,

174 whereas 11 patients were diagnosed as having ocular candidiasis

175 more than 8 days later (Figure 1). Twenty-one (38.9\%) patients

176 were diagnosed within 3 days, and the average time from a

177 positive blood culture to the diagnosis of ocular candidiasis was

1785.5 days. The time to the first negative fungal culture was

179 longer in the patients who were diagnosed with ocular candidiasis

180 at the time of a second fundoscopy performed more than 8 days

181 later after the positive fungal culture; all patients had

182 malignancies, had diabetes mellitus or were being treated with

183 immunosuppressive agents.

184 BDG values and ocular candidiasis

185 The diagnostic kit used for the measurement of BDG values

186 differed between KUH and Katsura; therefore, we created ROC

187 curves and determined that the appropriate cut-off values were

$188 \quad 22.5$ and 42.7 for KUH and Katsura, respectively. A case was

189 defined as BDG-high if the BDG value was higher than the cut-off

190 value. Using the cut-off value, more patients with ocular 
191 candidiasis than patients with non-ocular candidiasis were

192 grouped as BDG-high cases. There was no relationship between

193 the BDG value and causative agents (data not shown).

\section{Clinical outcome}

195 Among 54 cases of ocular candidiasis, 42 patients completed antifungal therapy without any worsening of visual acuity, and 12

197 patients died before the completion of antifungal therapy.

198 Among the chorioretinitis cases, 33 out of 35 patients who

199 provided a report indicated they had no ocular abnormalities.

200 Among the ocular candidiasis cases, micafungin was prescribed to

20123 patients, and fluconazole was prescribed to 25 patients. In 16

202 of the 23 patients who received micafungin therapy, the

203 antifungal treatment regimen was shifted to fluconazole or

204 amphotericin-B after the diagnosis of ocular candidiasis.

205 The 30-day mortality rate of patients with ocular abnormalities

206 was also higher, although these differences were not statistically

207 significant.

208 Analysis of risk factors

209 Candida albicans as the etiological agent $(\mathrm{P}=0.034 \mathrm{OR} ; 3.6895 \%$

210 CI 1.11-12.2) and higher beta-D-glucan values $(\mathrm{P}=0.001 \mathrm{OR} ; 9.99$ 
$21195 \%$ CI $2.60-21.3)$ were statistically significant for the risk

212 factors of ocular candidiasis, as determined by multivariate

213 regression analysis (Table 2).

215 Discussion

216 This study investigated the incidence and clinical characteristics

217 of ocular candidiasis. According to previous studies, the 218 prevalence of ocular candidiasis is estimated to be between 1 219 45\% [Rodrguez-Adria'n et al., 2003; Oude Lashof et al., 2011, 220 Parke et al., 1982; Brooks, 1989; Shah et al., 2008]. In this study, 221 ocular abnormalities occurred in $26 \%$ of 204 patients. It is likely 222 that patient selection led to the comparatively high prevalence of 223 ocular candidiasis. Among our patients, 50\% had malignancies, and more than $80 \%$ had predisposing risk factors such as antibiotic exposure, diabetes mellitus or the use of

226 immunosuppressive therapy. Furthermore, many patients had 227 been admitted for gastrointestinal diseases. Malignancy and 228 gastrointestinal disease were statistically significant risk factors 229 for ocular candidiasis as determined by chi-squared tests, 230 although the statistical significance was not retained in the 
231 multivariate regression model. Considering the pathogenesis of 232 endogenous ocular candidiasis, physical mucosal damage and 233 changes in normal flora induced by broad-spectrum antibiotics or 234 chemotherapy may facilitate the occurrence of ocular involvement.

235 Thus, the high prevalence of ocular candidiasis observed in this 236 study may have been the result of the severely 237 immunocompromised state of many patients.

239 have the greatest propensity to cause ocular candidiasis. In

240 contrast, C. parapsilosis was associated with ocular

241 manifestations significantly less frequently. In this study,

242 patients with ocular candidiasis were mostly infected with $C$.

243 albicans, a finding that is consistent with prior reports (Donahue

244 et al., 1994; Rodrguez-Adria'n et al., 2003; Oude Lashof et al.,

245 2011, Parke et al., 1982; Brooks, 1989; Shah et al., 2008). Some

246 of these cases occurred despite prompt catheter removal and the

247 immediate administration of antifungal agents after the onset of

248 Candida BSIs. These results suggest that fungal virulence as

249 well as host and treatment factors may be involved in the

250 pathogenesis of ocular candidiasis. It is likely that the high 
251 prevalence of $C$. albicans may also have increased the rate of 252 ocular candidiasis in this study.

253 Several studies revealed that the prospective evaluation of 254 circulating BDG in high-risk patients generates positive results

255 that are available before the culture results and can improve the 256 diagnosis of invasive candidiasis (Koo et al., 2009; Acosta et al., 257 2011; Ostrosky-Zeichner et al., 2005). In this study, more 258 patients with ocular candidiasis had higher BDG values, and 259 BDG positivity had a significant relationship with the 260 development of ocular candidiasis. However, there was no 261 relationship between elevated BDG values and etiologic agents 262 such as $C$. albicans or the prognosis of Candida BSIs (data not 263 shown). Although the BDG values that reflect the burden of 264 Candida species and the half-life are still unknown, when higher BDG values are present, ocular candidiasis may have already occurred in these patients, even if they are asymptomatic. Despite the high prevalence of ocular candidiasis, periodic ophthalmologic examinations are rarely performed in patients susceptible to opportunistic infection. According to the IDSA guidelines for invasive candidiasis, ophthalmologists should 
271 investigate each patient for the presence of ocular candidiasis

272 (Pappas et al., 2009), but the optimal timing for this evaluation

273 has not been established. Previous studies have advised an

274 interval of $<14$ days between the start of treatment and the first

275 retinal abnormality, an interval that is consistent with candidal

276 chorioretinitis (Rodrguez-Adria'n et al., 2003; Krishna et al.,

277 2000). Although the optimal treatment for endogenous ocular

278 candidiasis has not been clearly established yet, fluconazole and

279 voriconazole appear to be the most effective (pappas et al., 2009;

280 Khan et al., 2007). In our study, $80 \%$ of cases were diagnosed

281 within 7 days, and the antifungal agents were changed from

282 micafungin to azoles or amphotericin in 16 of the 42 ocular

283 candidiasis cases. If fundoscopy was performed later, the

opportunity for the earlier administration of potentially more

optimal antifungal agents might have been missed. In our study,

more than $80 \%$ of the ocular candidiasis cases were chorioretinitis,

287 which usually does not require surgical interventions. Many patients completed the course of antifungal therapy without any

289 visual disturbance. We speculate that earlier diagnosis and

290 treatment resulted in the improved prognosis regarding visual 
291 acuity. On the other hand, some ocular candidiasis cases were 292 diagnosed by a second fundoscopic examination more than 8 days 293 later. Ideally, when we consider a strategy based on the fact that 294 earlier diagnosis yields a better prognosis, fundoscopic 295 examination should be performed within first 7 days of antifungal 296 therapy, especially in those with $C$. albicans BSIs and higher BDG 297 values. In addition, follow-up fundoscopic examination should 298 also be considered in severely immunosuppressed patients, even 299 if the first fundoscopic examination yielded negative results.

\section{Study limitations}

302 This study has several limitations, including the fact that most of 303 the patients without ocular candidiasis were not re-examined serially. Conceivably, the disseminated fungal lesions could have arisen in healthy eyes after the initial exam and therefore may have been missed in some cases. Second, approximately $7.2 \%$ of the Candida BSI patients did not consult ophthalmologists for their underlying conditions. During discussion with those patients about the risk factors for ocular candidiasis, fundoscopy may have been indicated but not performed in some cases. 
311 Thirdly, we included the possible cases of ocular candidiasis who

312 had severe underlying diseases in this study. The prevalence

313 rate of ocular candidiasis might have been much lower than

314 reported here.

315 Transparency Declaration

316 This work was partly supported by grant H21-Shinko-Ippan-009

317 and H23-Shinkou-Ippan-018 from the Ministry of Health, Labor,

318 and Welfare of Japan. Some of the results were generated

319 during routine diagnostic activities. No commercial

320 relationships or potential conflicts of interest exist. 
1. Acosta J, Catalan M, Del Palacio-Perez-MEdel A, Montejo JC, De-La-Cruz-Bertolo J, Moraques MD, Ponton J, Finkelman MA, Del Placio A (2011) Prospective study in ciritcally ill non-neutropenic patients: diagnostic potential of (1,3)-8-D-glucan assay and circulating galactomannnan for the diagnosis of invasive fungal disease Eur J Clin Microbiol Infect Dis Aug 3. [Epub ahead of print]

2. Brooks RG (1989) Prospective study of Candida endophthalmitis in hospitalized patients with candidemia. Arch Intern Med 149:2226-8.

3. Donahue SP, Greven CM, Zuravleff JJ,Eller AW, Nguyen MH, Peacock JE Jr, Wagener MW, Yu VL (1994) Intraocular candidiasis in patients with candidemia. Clinical implications derived from a prospective multicenter study. Ophthalmology 101:1302-9.

4. Edmond MB, Wallace SE, McClish DK, Pfaller MA, Jones RN, Wenzel RP (1999) Nosocomial blood stream infections in United States hospitals: a three-year analysis. Clin Infect Dis 29: $239-44$. 
5. Jarvis WR (1995) Epidemiology of nosocomial fungal infections, with emphasis on Candida species Clin Infect Dis 20:1526-30.

6. Kao AS, Brandt ME, Pruitt WR, Conn LA, Perkins BA, Stephens DS, Baughman ES, Reingold AL, Rothrock GA, Pfaller MA, Pinner RW, Haijeh RA (1999) The epidemiology of candidemia in two United States cities: results of a population-based active surveillance Clin Infect Dis 29:1164-70.

7. Khan FA, Slain D, Khakoo RA (2007) Candida endophthalmitis: Focus on current and future antifungal treatment options. Pharmacotherapy 27:1711-21.

8. Koo S, Bryar JM, Page JH, Baden LR, Marty FM (2009) Diagnostic performance of the $(1 \rightarrow 3)-\beta-D-G l u c a n$ assay for invasive fungal disease. Clin Infect Dis 49:1650-9.

9. Krishna R, Amuh D, Lowder CY, Gordon SM, Adal KA, Hall G (2000) Should all patients with candidaemia have an ophthalmic examination to rule out ocular candidiasis? Eye $14: 30-34$.

10. Ostrosky-Zeichner L, Alexander BD, Kett DH, Vazquez J, 
Pappas PG, Saeki F, Ketchum PA, Wingard J, Schiff R, Tamura H, Finkelman MA, Rex JH (2005) Multicenter clinical evaluation of $(1 \rightarrow 3)$ - 6 -D-Glucan assay as an aid to diagnosis of fungal infections in humans. Clin Infect Dis 41:654-9.

11. Oude Lashof AM, Rothova A, Sobel JD Ruhnke M, Pappas PG, Viscoli C, Schlamm HT, Oborska IT, Rex JH, Kullberg BJ (2011) Ocular manifestations of candidemia. Clin Infect Dis $53: 262-8$

12. Pappas PG, Kauffman CA, Andes D, Benjamin DK Jr, Calandra TF, Edwards JE Jr, Filler SG, Fisher JF, Kullberg BJ, Ostrosky-Zeichner L, Reboli AC, Rex JH, Walsh TJ, Sobel JD; Infectious Diseases Society of America (2009) Clinical practice guidelines for the management of candidiasis: 2009 update by the Infectious Diseases Society of America. Clin Infect Dis 48:503-35.

13. Parke DW 2nd, Jones DB, Gentry LO (1982) Endogenous endophthalmitis among patients with candidemia. Ophthalmology 89:789-96.

14. Pfaller MA, and Diekema DJ (2007) Epidemiology of invasive candidiasis: a persistent public health problem Clin Microbiol 
$\operatorname{Rev} 20: 133-163$.

15. Rentz AM, Halpern MT, Bowden R (1998) The impact of candidemia on length of hospital stay, outcome, and overall cost of illness. Clin Infect Dis 27:781-788.

16. Rodrguez-Adria'n LJ, King RT, Tamayo-Derat LG, Miller JW, Garcia CA, Rex JH (2003) Retinal lesions as clues to disseminated bacterial and candidal infections: Frequency, natural history, and etiology. Medicine (Baltimore) $82: 187-202$.

17. Shah CP, Mckey J, Spirn MJ Maquire J (2008) Ocular candidiasis: a review. Br J Ophtalmol 92:466-468.

18. Sheng WH, Wang JT, Lu DC, Chie WC, Chen YC, Chang SC (2005) Comparative impact of hospital stay and outcome between community hospitals and medical centres. $J$ Hosp Infect 59:205-214.

19. Wisplinghoff H, Bischoff T, Tallent SM, Seifert H, Wenzel RP, and Edmond MB (2004) Nosocomial bloodstream infections in US hospitals: analysis of 24,179 cases from prospective nationwide surveillance study. Clin Infect Dis 39:309-317. 
402 Figure 1 Cumulative incidence of ocular candidiasis

403

404 Ocular candidiasis was diagnosed within 7 days after positive

405 blood culture in 43 patients, whereas 11 patients were diagnosed

406 with ocular candidiasis more than 8 days later.

407 


\section{Figure 1}

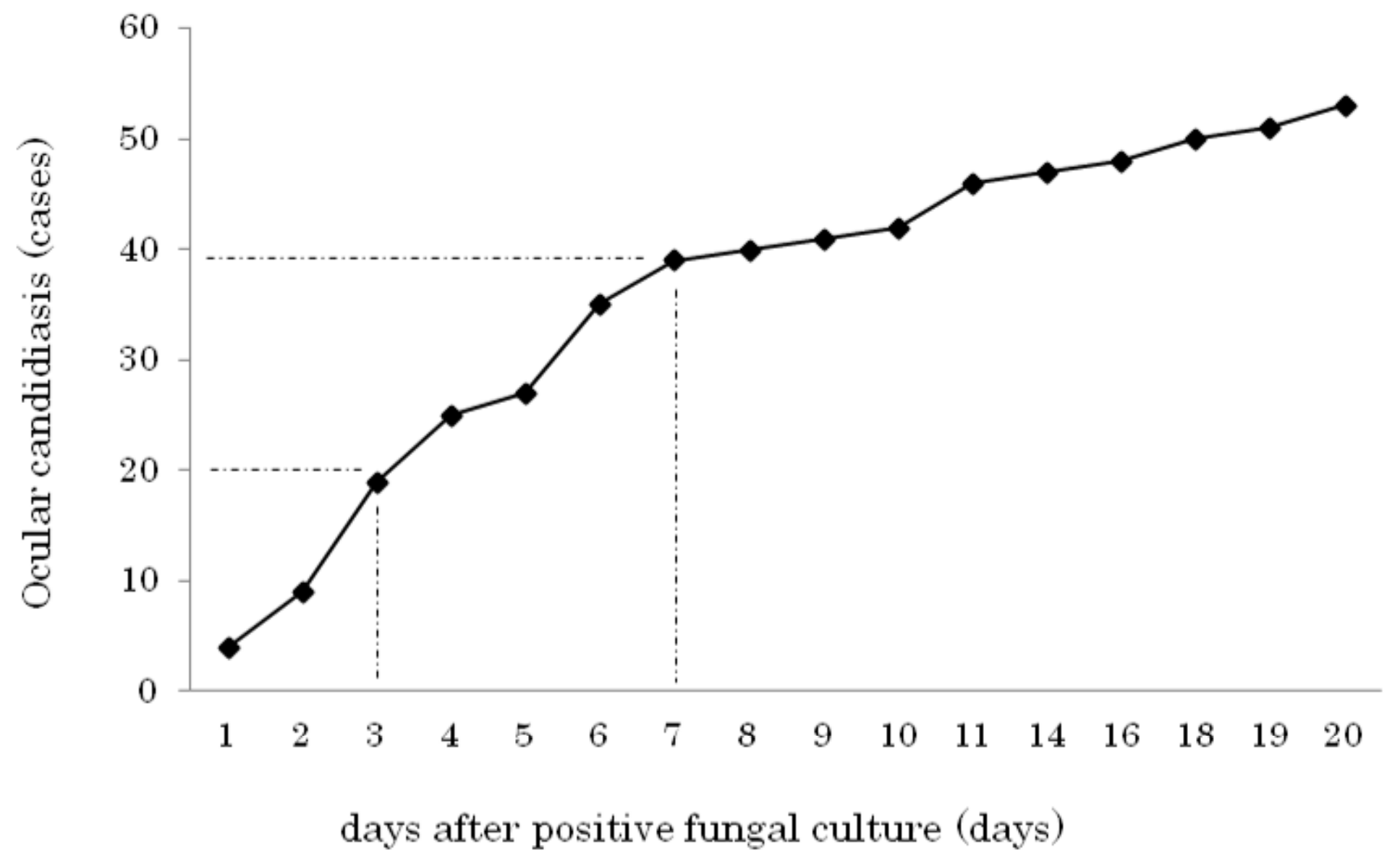


1 Table 1 Clinical characteristics of the study patients

\begin{tabular}{|c|c|c|c|c|c|}
\hline & $\begin{array}{c}\text { Ocular } \\
\text { candidiasis } \\
(\mathrm{N}=54)\end{array}$ & $(\%)$ & $\begin{array}{l}\text { Non-ocular } \\
\text { candidiasis } \\
\qquad(\mathrm{N}=150)\end{array}$ & $(\%)$ & $P$ \\
\hline Age & $\begin{array}{c}62.8 \pm \\
18.9\end{array}$ & & $63.14 \pm 19.8$ & & 0.923 \\
\hline Male & 28 & $51.9 \%$ & 80 & $53.3 \%$ & 0.875 \\
\hline Malignancy & 41 & $75.9 \%$ & 60 & $40.0 \%$ & $<0.001$ \\
\hline Diabetes mellitus & 12 & $28.6 \%$ & 22 & $17.3 \%$ & 0.210 \\
\hline Digestive tract involvement & 35 & $68.6 \%$ & 61 & $41.5 \%$ & 0.001 \\
\hline Immunosuppressive agent & 20 & $37.0 \%$ & 32 & $21.3 \%$ & 0.083 \\
\hline Antibiotic within one month & 46 & $86.8 \%$ & 112 & $74.7 \%$ & 0.083 \\
\hline Surgery within one month & 17 & $31.5 \%$ & 46 & $30.7 \%$ & 0.911 \\
\hline C. parapsilosis & 3 & $5.6 \%$ & 35 & $23.3 \%$ & 0.002 \\
\hline C. albicans & 40 & $74.1 \%$ & 67 & $44.7 \%$ & $<0.001$ \\
\hline C. glabrata & 5 & $9.3 \%$ & 19 & $12.7 \%$ & 0.626 \\
\hline C. tropicalis & 5 & $9.3 \%$ & 18 & $12.0 \%$ & 0.862 \\
\hline High beta-D-glucan $(\mathrm{N}=88)$ & 29 & $74.4 \%$ & 31 & $34.4 \%$ & $<0.001$ \\
\hline $\begin{array}{l}\text { Time to first negative blood } \\
\text { culture, mean, range (days) }\end{array}$ & $\begin{array}{c}5.52 \pm \\
4.04,1-14\end{array}$ & & $\begin{array}{c}5.32 \pm 3.40 \\
1-27\end{array}$ & & 0.787 \\
\hline $\begin{array}{l}\text { Blood culture to antifungal } \\
\text { agent, mean, range (days) }\end{array}$ & $\begin{array}{c}1.82 \pm \\
1.37,1^{-5}\end{array}$ & & $\begin{array}{c}2.34 \pm 2.81 \\
1-5\end{array}$ & & 0.117 \\
\hline $\begin{array}{l}\text { First sign of infection to } \\
\text { removal of the catheter, }\end{array}$ & $\begin{array}{c}1.52 \pm \\
2.30,1-12\end{array}$ & & $\begin{array}{c}1.56 \pm 2.17 \\
1-11\end{array}$ & & 0.920 \\
\hline
\end{tabular}


mean, range (days)

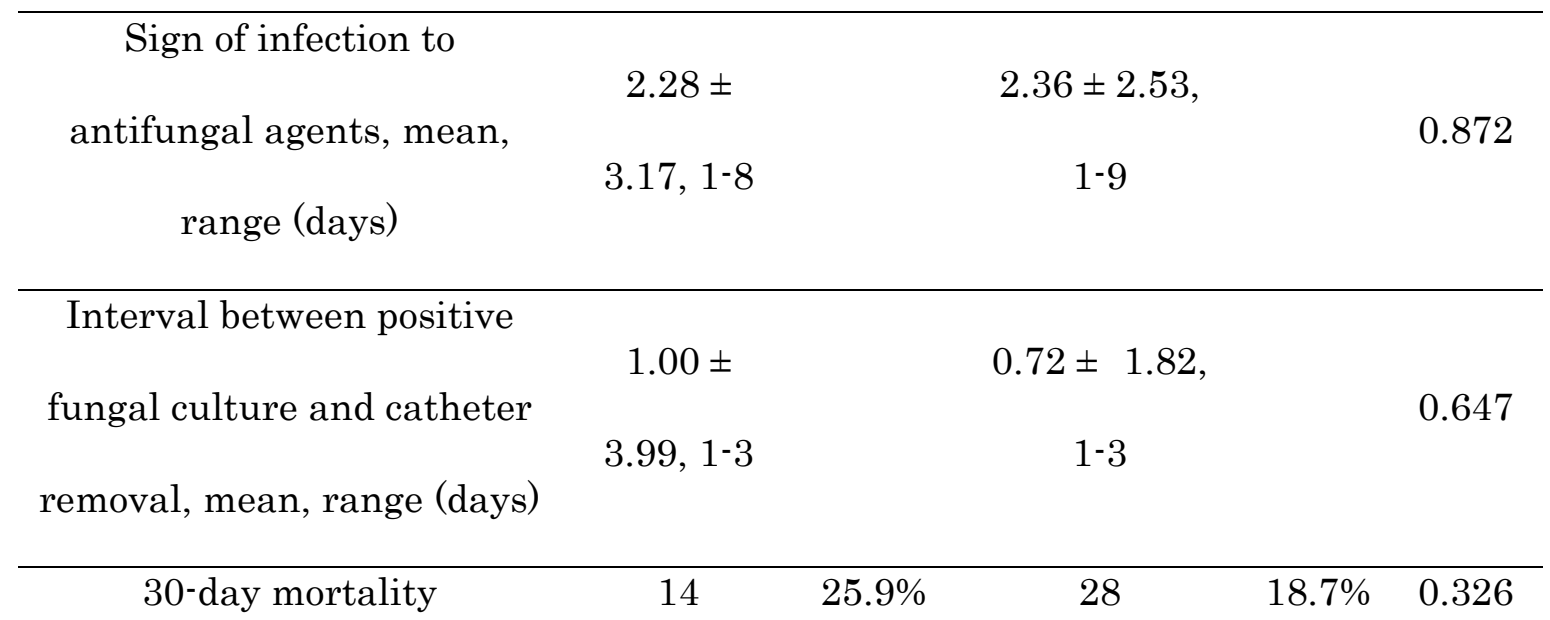


Table 2 Results of multilvariate regression analysis of factors associated with ocular candidiasis

\begin{tabular}{cccc} 
& $P$ value & $\operatorname{Exp}(\mathrm{B})$ & $95 \% \mathrm{CI}$ \\
\hline (1,3)-B-D-glucan high & 0.001 & 9.99 & $2.60-21.3$ \\
\hline C. albicans & 0.034 & 3.68 & $1.11-12.2$ \\
\hline Digestive tract involvement & 0.290 & & \\
\hline Malignancy & 0.714 & \\
\hline Immunosuppressive agent & 0.625 & \\
\hline Antibiotic within one month & 0.483 & \\
\hline
\end{tabular}

